\title{
Observations on the warm up phenomenon in angina pectoris
}

\author{
M JOY, A W CAIRNS, D SPRIGINGS \\ From St Peter's Hospital, Chertsey, Surrey
}

SUMMARY Adaptation to exercise was investigated in 14 men aged 34-69 years (mean 51) with stable exertional angina caused by occlusive coronary artery disease. All underwent exercise electrocardiography to symptom limitation according to the Bruce protocol (first effort), and exercise to the onset of angina (warm up) followed by four minutes' rest, followed by exercise to symptom limitation (second effort). This protocol was repeated after sequential treatment for one month each with nifedipine $10 \mathrm{mg}$ three times a day and with timolol $10 \mathrm{mg}$ twice a day. Warm up significantly increased walking time to the onset of angina by $34.5 \%$ and to maximal exercise by $29.5 \%$. The heart rate and rate-pressure product were significantly higher on second effort both at the onset of angina (by $7.0 \%$ and $11.1 \%$ respectively) and at maximal exercise (by $10.5 \%$ and $15.4 \%$ respectively). ST segment displacement was not significantly different after warm up. The effect of warm up on walking time to the onset of angina was markedly reduced after treatment with nifedipine but little influenced by timolol. Mean (SE) walking time after warm up on no treatment was $10.1(0.7) \mathrm{min}$; after treatment with nifedipine it was $10.0(0.6)$ min and after treatment with timolol it was $9.7(0.4) \mathrm{min}$.

These data demonstrate a substantial improvement in exercise performance after warm up and are consistent with the hypothesis that submaximal exercise in angina pectoris facilitates myocardial oxygen uptake by coronary vasodilatation.

Heberden first described the loss of symptoms with continued exercise in a patient experiencing angina. ${ }^{2}$ It has been variously described; by Osler as "walking through" 3 and by Wenckebach as the "second wind". 4 Gallavardin recorded the "warming up" effect of previous exercise in angina pectoris 5 and Price described the phenomenon as "first effort angina". 6 Gallavardin claimed it was not an uncommon phenomenon ${ }^{5}$ but others have been unable to demonstrate it. ${ }^{7}$ Wayne and Gaybriel described two patients whose exercise performance was enhanced when they re-exercised between five and 30 minutes after an attack of angina, the effect being lost when the interval was one hour. ${ }^{8}$ Exercise performance

A preliminary report of this work was presented to the Third Joint Meeting of the Working Groups of the European Society of Cardiology, Spa, Belgium 1983. ${ }^{1}$

Requests for reprints to Dr M Joy, St Peter's Hospital, Guildford Road, Chertsey, Surrey KT16 OPZ.

Accepted for publication 19 February 1987 was reduced when the patient re-exercised within two minutes of having symptoms.

Although the effects of physical training on exercise performance in angina pectoris have received attention $^{910}$ studies of short term adaptation to exercise are limited..$^{81112}$

\section{Patients and methods}

\section{PATIENTS}

We studied 14 white men aged 34-68 (mean 51) with $a \geqslant 6$ month history of stable exertional angina. All were normotensive (blood pressure $<160 / 95 \mathrm{~mm} \mathrm{Hg}$ ), and had no past history of myocardial infarction and no evidence of valvar heart disease. They also had a normal chest radiograph and resting electrocardiograph, but planar or downsloping ST segment depression of $\geqslant 1 \mathrm{~mm}$ developed when they exercised according to the Bruce treadmill protocol. In all of them characteristic defects of thallium-201 myocardial imaging developed during exercise and coronary artery disease 
was confirmed by arteriography. Informed signed consent was obtained from all participants. The investigation had the approval of the hospital ethics committee.

\section{METHODS}

All patients performed the following tests on different days in random order while on no treatment (the Bruce treadmill protocol was used): (a) maximal exercise electrocardiography (first effort); (b) exercise to the onset of angina (warm up) followed by four minutes' rest followed by maximal exercise (second effort). The walking time to the onset of angina and to maximal exercise were recorded, together with heart rate (from the electrocardiograph) and systolic blood pressure (obtained with a sphygmomanometer).

After an interval of 2-4 weeks all patients were randomised into one of two groups; the first was treated with timolol $10 \mathrm{mg}$ twice a day and the second with nifedipine $10 \mathrm{mg}$ three times a day. The two exercise protocols were repeated after four weeks and the patients crossed over; the protocol was repeated at the end of a further four weeks. Nifedipine was given 30 minutes before exercise and timolol 60 minutes beforehand. Patients could take sublingual glyceryl trinitrate for symptomatic relief during the period of the study but not on the day of investigation. No other medication was taken. All recordings were made at the same time of day to exclude any possible circadian influence. ${ }^{13}$ The mean (SD) temperature of the exercise laboratory was maintained at $20(1)^{\circ} \mathrm{C}$.

Exercise electrocardiography was performed on an Avionics A10 treadmill in conjunction with an Avionics 3000 three channel oscillographic monitor. Leads CM4, CM5, and CM6 were recorded for 5-10 seconds at rest, after each minute of exercise, at the end of exercise, and for each of 10 minutes of recovery. The treadmill and chart speeds were regularly checked and the recording apparatus was optimally damped. ST segment displacement $60 \mathrm{~ms}$ after the nadir of the $S$ wave was computed and checked against the chart; the lead showing the greatest displacement at maximal exercise was chosen. We calculated the product of heart rate and systolic blood pressure.

We calculated the means and standard errors of the paired observations (on first and second efforts) and tested their difference from zero by Student's paired $t$ test.

\section{Results}

All 14 men had important coronary artery disease (defined as a stenosis $>50 \%$ in the left main artery or $>70 \%$ in other major arteries). Ten subsequently underwent coronary artery bypass grafting. Left ventricular end diastolic pressure was normal in all but three in whom it was slightly raised $(<18 \mathrm{~mm} \mathrm{Hg}$ ). Regional hypokinesis was commonly found on left ventricular angiography but none of the patients showed more severe wall motion abnormalities.

\section{PATIENTS NOT ON DRUG TREATMENT}

Figs 1 and 2 show the mean walking times and the effect of warm up both on the onset of angina and maximal exercise. Walking time to the onset of angina was improved by $34.5 \%(p<0.001)$ and to maximal exercise by $29.5 \%(p<0.001)$. Figs 3 and 4 show the effect of warm up on the heart rate at the onset of angina and at maximal exercise. After warm up the heart rate at the onset of angina was increased by $7.0 \%(p<0.01)$ and at maximal exercise by $10.5 \%(p<0.01)$. Similarly, the rate-pressure product at the onset of angina and at maximal exercise was improved after warm up by $11 \cdot 1 \%$ ( $p<$ $0.01)$ and $15.4 \%(p<0.02)$ respectively (figs 5 and 6). ST segment displacements were not significantly different at the onset of angina before and after warm up $(p>0.05)$ or at maximal exercise before and after warm up ( $p>0.05)$. There was no significant difference between the heart rate or ratepressure product at the onset of angina on first effort and at the same walking time on second effort ( $p>$ $0.47, p>0.15$ ) (figs 7 and 8). In contrast, both the heart rate and rate-pressure product were higher $(p<0.025 ; p<0.05)$ on second effort at the walking time of maximal exercise than on first effort (figs 7 and 8). ST segment depression was less on second

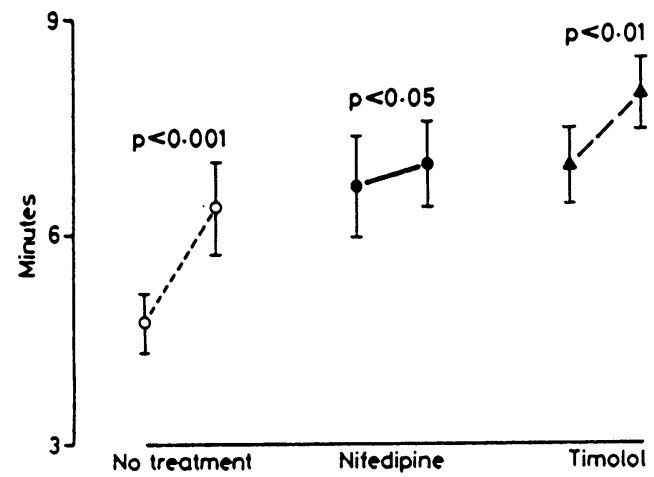

Fig 1 Walking time to the onset of angina on first and second efforts (left and right hand data points respectively) on no treatment and after treatment for one month with nifedipine $10 \mathrm{mg}$ three times a day and with timolol $10 \mathrm{mg}$ twice a day. In.this and subsequent figures the data points are group mean values and the vertical bars are 1 SE. 


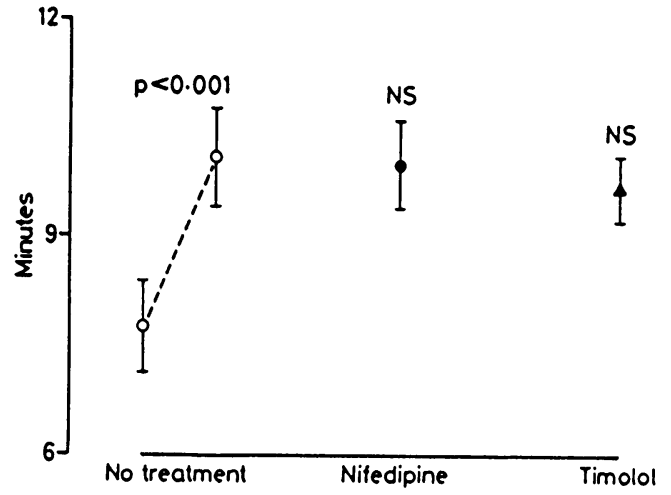

Fig 2 Maximal walking time on first and second efforts on no treatment (left and right hand data points respectively) and on second effort after treatment with nifedipine and with timolol.

effort both at the point of onset of angina and at the walking time of maximal exercise on first effort $(p<$ 0.025 ; $<<0.01$ ) (fig 9).

\section{EFFECT OF NIFEDIPINE}

Treatment with nifedipine increased the mean walking time to the onset of angina on first effort by $40.6 \%(\mathrm{p}<0.01)$ (fig 1$)$. Warm up increased the walking time to the onset of angina by a further $4.0 \%(\mathrm{p}<0.05)$. The heart rate and rate-pressure product at the onset of angina increased by $2.4 \%$ and $4.0 \%$ respectively $(p<0.01, p<0.025)$ (figs 3 and 5 ). On second effort, maximal walking time, heart rate, rate-pressure product, and ST segment depression did not differ significantly from values seen on no treatment after warm up ( $p>0.40, p>$

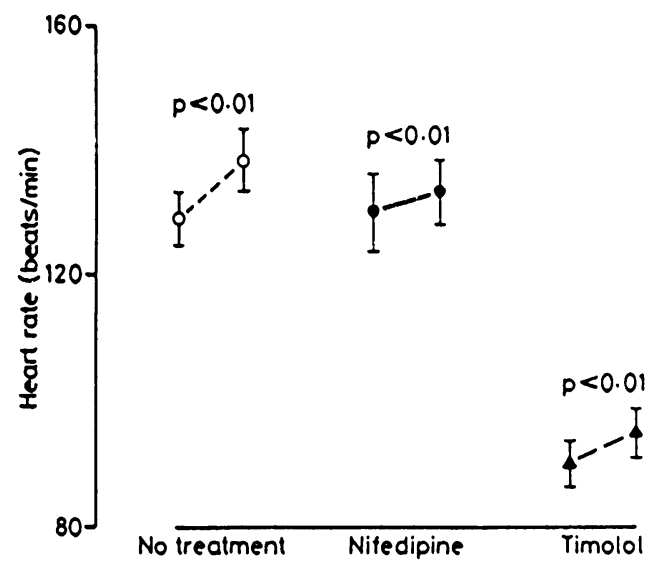

Fig 3 Heart rate at the onset of angina on first and second efforts (left and right hand data points respectively) on no treatment and after treatment with nifedipine and with timolol.

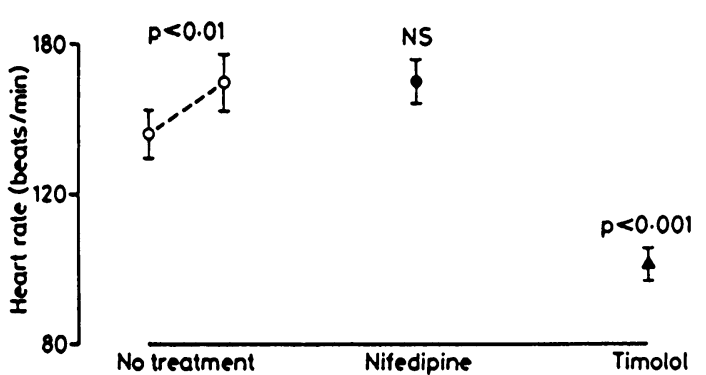

Fig 4 Heart rate at maximal exercise on first and second efforts on no treatment (left and right hand data points respectively) and on second effort after treatment with nifedipine and with timolol.

$0 \cdot 15, p>0 \cdot 15$, and $p>0.05$ respectively) (figs 2,4 , and 6).

\section{EFFECT OF TIMOLOL}

As would be expected, timolol reduced the heart rate and rate-pressure product both on first and second efforts (figs 3 to 6). Treatment with timolol increased the walking time to the onset of angina on first effort by $46.9 \%$ (p < 0.001) (fig 1). Warm up further increased the walking time to the onset of angina by $14.7 \%(p<0.01)$. The heart rate and rate-pressure product at the onset of angina were increased by $5.5 \%$ and $10.0 \%$ respectively on second effort ( $p<0.01 ; p<0.005)$ (figs 3 and 5). However, maximal walking time on second effort was not significantly different from that achieved on no treatment $(p>0.20)$ (fig 2). On second effort the heart rate and rate-pressure product at maximal exercise were significantly lower on timolol than on no treatment ( $p<0.001 ; p<0.001$ ) (figs 4 and 6 ). There was also less ST segment displacement than on no treatment $(p<0.01)$ (fig 9$)$.

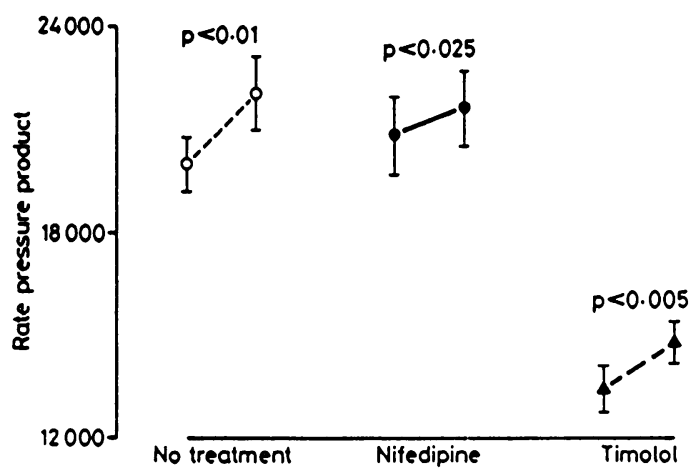

Fig 5 Rate-pressure product ( $\mathrm{mm} \mathrm{Hg} \times$ beats/min) at the onset of angina on first and second efforts (left and right hand data points respectively) on no treatment and after treatment with nifedipine and with timolol. 


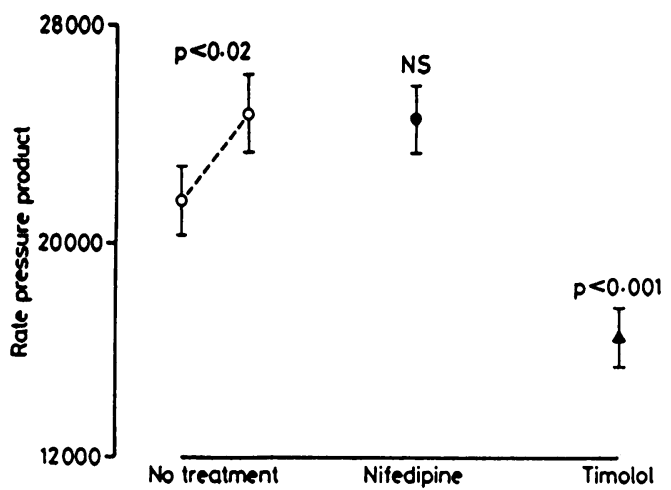

Fig 6 Rate-pressure product ( $\mathrm{mm} \mathrm{Hg} \times$ beats $/ \mathrm{min})$ at maximal exercise on first and second efforts on no treatment (left and right hand data points respectively) and on second effort after treatment with nifedipine and with timolol.

\section{Discussion}

In previous studies of patients with stable exertional angina performance did not improve on repeated exercise testing after 10 to 15 minutes' recovery, ${ }^{14-16}$ and a pause of $1-2$ minutes also had no beneficial effect. ${ }^{7}$ By contrast, in our subjects walking time was substantially improved when a first effort to the point of angina was followed by four minutes' recovery. Heart rates and rate-pressure products were also higher on second effort, suggesting increased myocardial oxygen consumption. ${ }^{17}$ As the degree of ST segment depression was not significantly different, either at the point of angina or on maximal exercise before or

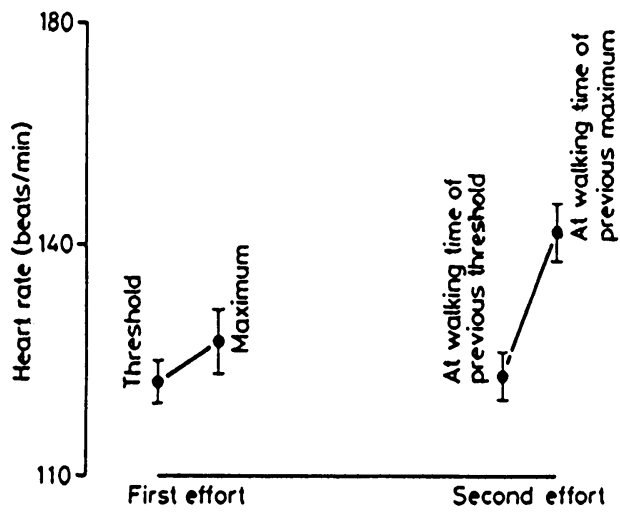

Fig 7 The heart rate on no treatment at the threshold for angina at first effort was similar to the heart rate at the same walking time on second effort $(p>0.47)$. The heart rate was significantly increased on second effort at the maximum walking time achieved during first effort $(p<0.025)$.

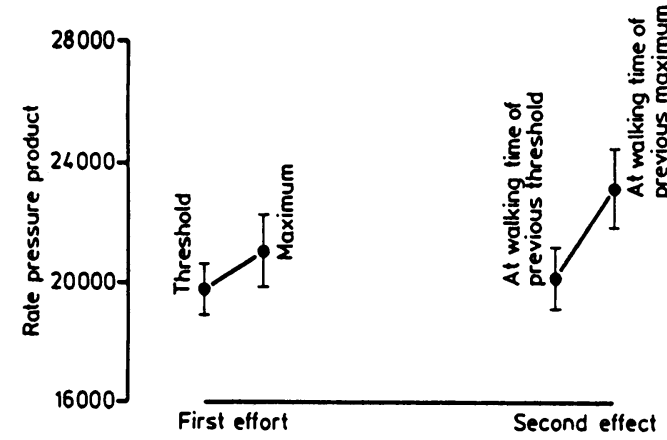

Fig 8 The rate-pressure product ( $\mathrm{mm} \mathrm{Hg} \times$ beats/min) on no treatment at the threshold for angina on first effort was similar to the product at the same walking time on second effort ( $p>0 \cdot 15)$. On second effort the rate-pressure product at the maximum walking time achieved during the first effort was increased $(p<0.05)$.

after warm up, it may be inferred that the increase in heart work was not accompanied by increased myocardial ischaemia. ${ }^{14}$ Coronary blood flow is closely related to myocardial oxygen demand ${ }^{18}$ and coronary reserve is progressively diminished by stenosis of a major coronary vessel. ${ }^{19}$ Slight variation in the calibre of a stenosed vessel has a major effect on blood flow ${ }^{20}$ and may explain the variable exercise performance of patients with vasospastic angina. ${ }^{21}$ A metabolically mediated vasodilatation of stenosed epicardial arteries could provide one explanation for our observations.

Nifedipine largely abolished the effect of warm up, and walking time to angina on second effort was increased by only $4 \%$. Yet at maximal walking time, heart rate and rate-pressure product were not significantly different from those achieved without medication after warm up; the favourable action of

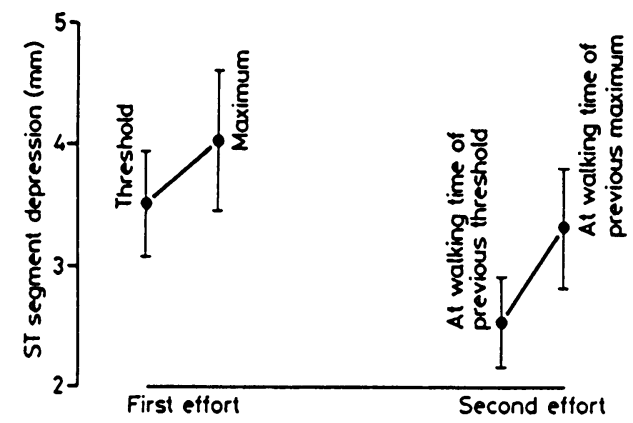

Fig $9 S T$ segment depression ( $\mathrm{mm}$ ) on no treatment at the threshold for angina on first effort was greater than at the same walking time on second effort $(p<0.025)$. ST segment depression at maximal exercise on first effort was also greater than that at the same walking time on second effort $(p<0.01)$. 
nifedipine and the warm up effect may thus share a common mechanism. The pharmacodynamic effects of nifedipine are several and their net haemodynamic result is modified by peripheral circulatory reflex activity. Systemic administration of nifedipine at rest leads to a fall in total peripheral resistance and in coronary vascular resistance, with an increase in coronary blood flow. The reduction in afterload is to some extent buffered by reflex tachycardia and peripheral vasoconstriction. ${ }^{22}$ Early exercise data suggested that nifedipine enhanced exercise capacity by a relative reduction in myocardial oxygen uptake (expressed by a lack of increase in the double product with increased performance) rather than by an increase (which would have been expressed by an increase in the double product). ${ }^{23}$ After warm up, however, we found an increase in the double product in patients on nifedipine. Other investigators have suggested that the most important net effects include a decrease in coronary vascular resistance, ${ }^{24}$ a reduction in the arteriocoronary sinus oxygen difference, ${ }^{25}$ and an increase in coronary blood flow. ${ }^{26}$ Nifedipine does not alter the filling pressure in normal left ventricular function. ${ }^{27}$

Reduction of myocardial oxygen demand is the major antianginal effect of $\beta$ adrenoceptor blocking agents, including timolol. ${ }^{28}{ }^{29}$ As such agents may increase $\alpha$ mediated vasoconstriction of coronary vessels, ${ }^{30}$ coronary vasodilatation, by whatever means, should enhance exercise performance. Our results accord with this: the warm up effect was not attenuated in subjects on timolol and walking time to the onset of angina on second effort was increased by $15 \%$. Had a reduction in preload, afterload, or both been the explanation for the enhanced walking time in our patients after warm up, then the rate-pressure product (reflecting myocardial oxygen uptake) should not have increased significantly.

There has been some speculation about the possible mechanisms of the warm up effect. ${ }^{63132}$ There is a near constant rate-pressure product at the threshold of angina ${ }^{1433} 34$ and variation in symptoms may be accounted for by variation in the circulatory responses. ${ }^{14}$ Robinson, however, did not observe a warm up effect, possibly because his protocol allowed 10 to 15 minutes' recovery between exercise trips. ${ }^{14}$ Although we did not investigate this point systematically, it seems likely that the warm up effect is of short duration only. We chose a four minute recovery period for our protocol because it reflected the patients' experience.

Other determinants of myocardial oxygen consumption need to be considered in the context of the warm up effect. A reduction in wall tension due to reduced left ventricular end diastolic pressure caused by a fluid shift or vasodilatation may be a factor. ${ }^{35}$ One study of supine bicycle exercise, however, demonstrated that although left ventricular end diastolic pressure was reduced during a second period of exercise 25 minutes after exercising to angina, mean values for exercise time, rate-pressure product, and ST segment depression at the onset of angina were not significantly different. ${ }^{36}$ Our data do not exclude a contribution to the effect by this mechanism.

A recent study of patients with exertional angina who had isolated disease of the left anterior descending coronary artery showed that myocardial ischaemia was less during the second of two identical periods of pacing stress separated by 5-15 minutes. ${ }^{37}$ Peak coronary flow, however, was not significantly different during the second period but regional myocardial oxygen consumption was reduced; the authors attributed this result to an ischaemic fall in contractility. Although this result does not support the suggestion that the warm up effect is due to coronary vasodilatation, it should be noted that their patients were not paced to a high rate-pressure product or to the same degree of ischaemia on the second occasion.

The warm up effect may be demonstrated objectively in the exercise laboratory, although its exact time course has yet to be defined. Our observations seem to favour coronary vasodilatation as the mechanism for this effect. It is not known whether a local metabolic, hormonal, or neurogenic pathway is responsible. The possibility of a contribution from a reflex reduction in afterload cannot, however, be discounted. Given the similarities between the warm up effect and treatment with nifedipine, it is noteworthy that nifedipine has been reported to be effective in relieving ischaemia only in myocardium supplied by a stenosed (but not occluded) vessel. ${ }^{38}$ The relation between the warm up effect and coronary anatomy remains to be established.

\section{References}

1 Joy M, Cairns A, Shah S. Observations on the second wind phenomenon. Eur Heart $J$ 1983;4 (suppl E):24.

2 Heberden W. A letter to Dr Heberden concerning the angina pectoris and an account of the dissection of one who had been troubled with that disorder. Medical Transactions published by the College of Physicians, London 1785;3:1-11.

3 Osler W. Lectures on angina pectoris and allied states. New York: D Appleton, 1897:52.

4 Wenckebach KF. Toter Punkt, second wind und angina pectoris. Wien Klin Wochenschr 1928;41:1-10.

5 Gallavardin L. Les angines de poitrine. Paris: Masson \& Cie, 1925:119.

6 Price RK. First effort angina. Second wind in angina pectoris. Br Heart J 1951;13:197-202 
7 Wayne EJ, LaPlace LB. Observations on angina of effort. Clin Sci 1933;1:103-29.

8 Wayne EJ, Gaybriel A. Observations on the effect of food, gastric distension, external temperature and repeated exercise on angina of effort with a note on angina sine dolore. Clin Sci 1934;1:287-304.

9 Detry JM, Bruce RA. Effects of physical training on exertional ST segment depression in coronary heart disease. Circulation 1971;44:390-7.

10 Sim DN, Neill WA. Investigation of the physiological basis for increased exercise threshold for angina pectoris after physical conditioning. J Clin Invest 1974;54:763-70.

11 Patterson JE, Clarke TW, Levy RL. A comparison of electrocardiographic changes observed during the anoxaemia test on normal persons and on patients with coronary sclerosis. Am Heart $J$ 1942;23:837-46.

12 MacAlpin RN, Kattus AA. Adaptation to exercise in angina pectoris. The electrocardiogram during treadmill walking and coronary angiographic findings. Circulation 1966;33:183-201.

13 Joy $M$, Pollard CM, Nunan TO. Diurnal variation in exercise responses in angina pectoris. $\mathrm{Br}$ Heart $\mathrm{J}$ 1982;48:156-60.

14 Robinson BF. Relation of heart rate and systolic blood pressure to the onset of pain in angina pectoris. Circulation 1969;35:1073-83.

15 Redwood DR, Rosing DR, Goldstein RE, Beiser GD, Epstein SE. Importance of the design of an exercise protocol in the evaluation of patients with angina pectoris. Circulation 1971;43:618-28.

16 Kattus AA, Alvaro AA, Zohman LR, Coulson AH. Comparison of placebo, nitroglycerin, and isosorbide dinitrate for effectiveness of relief of angina and duration of action. Chest 1979;75:17-23.

17 Gobel FL, Nordstrom LA, Nelson RR, Jorgensen CR, Wang $Y$. The rate-pressure product as an index of myocardial oxygen consumption during exercise in patients with angina pectoris. Circulation 1978; 57:549-56.

18 Feigl EO. Coronary physiology. Physiol Rev 1983; 63:1-205.

19 Gould KL, Lipscomb K. Effects of coronary stenoses on coronary flow reserve and resistance. Am J Cardiol 1974;34:48-55.

20 Epstein SE, Talbot TL. Dynamic coronary tone in precipitation, exacerbation and relief of angina pectoris. Am J Cardiol 1981;48:797-803.

21 DeServi S, Specchia G, Falcone C, et al. Variable threshold exertional angina in patients with transient vasospastic myocardial ischaemia. Repeat exercise test results and therapeutic implications. Am J Cardiol 1983;51:397-402.

22 Stone PH, Antman EM, Muller JE, Braunwald E. Calcium channel blocking agents in the treatment of cardiovascular disorders. Part II Haemodynamic effects and clinical applications. Ann Intern Med 1980; 93:896-904.
23 Moskowitz RM, Piccini PA, Nacarell G, Zelis R. Nifedipine therapy for stable angina pectoris: preliminary results of effects on angina frequency and treadmill exercise response. Am J Cardiol 1979; 44:811-6.

24 Bagger JP, Nielsen TT. Influence of nifedipine on coronary haemodynamics and myocardial metabolism in coronary disease. Eur Heart $J$ 1985;6:75-84.

25 Stone D. The effects of nifedipine on coronary haemodynamics and exercise left ventricular function. Postgrad Med J 1983;59 (suppl 2):25-38.

26 Specchia G, DeServi S, Falcone C, et al. Effects of nifedipine on coronary haemodynamic findings during exercise in patients with stable exertional angina. Circulation 1983;68:1035-43.

27 Kurnik PB, Tiefenbrunn AJ, Ludbrook PA. The dependence of the cardiac effects of nifedipine on the responses of the peripheral vascular system. Circulation 1984;69:963-72.

28 Thadani U, Sharma B, Meeran MK, Majid PA, Whitaker W, Taylor SH. Comparison of adrenergic beta-receptor antagonists in angina pectoris. $\mathrm{Br} \mathrm{Med}$ $J$ 1973;i:138-42.

29 Harris FJ, Low RI, Paumer L, Amsterdam EA, Mason DT. Anti anginal efficacy and improved exercise performance with timolol. Twice-daily beta-blockade in ischaemic heart disease. Am J Cardiol 1983;51:13-8.

30 Kern MJ, Ganz P, Horowitz JD, et al. Potentiation of coronary vasoconstriction by beta adrenergic blockade. Circulation 1983;67:1178-85.

31 MacAlpin RN, Kattus AA. Adaptation of exercise in angina pectoris. The electrocardiogram during treadmill walking and coronary angiographic findings. Circulation 1966;33:183-201.

32 Jaffe MD, Quinn NK. Warm up phenomenon in angina pectoris. Lancet 1980;ii:934-6.

33 Fabián J, Stolz I, Janota M, Roháč J. Reproducibility of exercise tests in patients with symptomatic ischaemic heart disease. Br Heart J 1975;37:785-9.

34 Starling MR, Moody M, Crawford MH, Levi B, O'Rourke RA. Repeat exercise treadmill testing: variability of results in patients with angina pectoris. Am Heart J 1984;107:298-303.

35 Rubenberg HL. Of pre-loads, after-loads and warm ups [Letter]. Lancet 1981;i:272.

36 Thadani U, Lewis JR, Manyai D, et al. Are the clinical and haemodynamic events during exercise stress testing in invasive studies in patients with angina pectoris reproducible? Circulation 1980;61:744-50

37 Williams DO, Bass TA, Gewirtz H, Most AS. Adaptation to the stress of tachycardia in patients with coronary artery disease. Insight into the mechanism of the warm-up phenomenon. Circulation 1985;71: 687-92.

38 Shulz W, Jost S, Kober G, Kaltenbach M. Relation of antianginal efficacy of nifedipine to degree of coronary arterial narrowing and to presence of coronary collateral vessels. Am J Cardiol 1985;55:26-32. 